\title{
Experimental results using corona discharge to attempt to reproduce the Turin Shroud Image
}

\author{
Giulio Fanti1 ${ }^{1, a}$, Francesco Lattarulo ${ }^{2, b}$, and Giancarlo Pesavento ${ }^{3, \mathrm{c}}$ \\ ${ }^{1}$ Department of Industrial Engineering, University of Padova, Padova, Italy \\ ${ }^{2}$ Department of Electrical and Information Engineering Technical University of Bari, Bari, Italy \\ ${ }^{3}$ Department of Industrial Engineering University of Padova, Padova, Italy
}

\begin{abstract}
It is well known that the Turin Shroud (TS) body image is not reproducible with all its peculiar features, but a great number of attempts have been made to reproduce at the same time the macro- and micro-scale properties of the double (front and back) image of the human body impressed on the most important Relic of the Christianity. The authors are convinced that among these experiments, those performed using CD (Corona Discharge) allowed to reproduce the greatest number of similarities.

The experiments were performed in the high-voltage laboratory of the University of Padua where CD was used to produce human images using linen clothes enveloping a halfscale conductive manikin of a man postured in the same way as the TS Man.

The manikin was suspended from ground and connected through the feet to a $300-\mathrm{kV}$ DC supply. The resulting CD produced a human image on the enveloping linen cloth after proper aging. Due to the draping configuration, the image showed various distortions of pseudo-cylindrical type.

The structure of the resulting electric field and the influence of the cover producing a partial barrier effect was studied together with the pseudo-cylindrical distortions that resulted less evident in the TS image. This information was used to study the enveloping configuration of the TS during the image formation
\end{abstract}

\section{Introduction}

The Turin Shroud (TS) is a linen sheet, $4.4 \mathrm{~m}$ long and $1.1 \mathrm{~m}$ wide, that enveloped the corpse of a scourged, thorn-crowned, and crucified man stabbed in the side by an object similar to a lance [1, 2]. There are other marks, overlying the double, front and back body image, like those caused by blood, fire, water, and folding that are partially superimposed to the image.

The TS is believed by many to be the burial cloth in which Jesus Christ was enveloped and placed in a Palestine tomb about 2000 years ago, but some still declare that it is a fake. Therefore it represents the most important but also controversial Relic of Christianity.

\footnotetext{
a e-mail: giulio.fanti@unipd.it

b e-mail: francesco. lattarulo@poliba.it

c e-mail: giancarlo.pesavento@unipd.it
}

This is an Open Access article distributed under the terms of the Creative Commons Attribution License 4.0, which permits unrestricted use, distribution, and reproduction in any medium, provided the original work is properly cited. 
The interest of the investigators largely increased after the photographs made by Pia in 1898, when the body image was interpreted as a photographic negative [3]. This observation introduced a new age of studies resulting in a lively production of scientific papers [1, 4].

The mapping of the luminance levels relative to the body image, if correlated to the body-cloth distance, revealed a 3D shape of the corresponding human surfaces [5].

The most wide and detailed scientific analysis of the TS was performed in 1978 by the STURP (Shroud of TUrin Research Project) [1, 4]. In spite of the great number of facts detected by such a Project, the arguments furnished to explain the body image formation resulted unsatisfactory, a lack that extends to nowadays and pushed some researchers to name it as an "impossible image" [6].

The TS body image is superficial both at macroscopic and at microscopic level [7] and in some areas of the frontal image, such as those of the face and perhaps the hands, it appears superficial on both sides [8]. Many other particular features impossible to be reproduced all together are reported in the literature $[9,10]$.

Hypotheses about nature of the CD factors for the TS imaging have been described in Refs. [11-13], where experimental data in terms of microscopic features are also discussed. However, the experimental efforts made to analyze macroscopic features are quite rare $[14,15]$.

The present paper points to show and discuss the experimental images, visible at naked eye, formed on linen sheets exposed to $\mathrm{CD}$. This material was used to cover a half-size scale conducting manikin raised to a DC potential up to $300 \mathrm{kV}$. As the frontal image of the TS is very similar to the dorsal one, the attempt of the authors to reproduce the TS image is for the moment only addressed to the frontal image; future test will also consider the dorsal one.

\section{Experimental setup}

In the present paper any reference to the fact that the TS enveloped the body of Jesus Christ and that it was put in a rocky sepulcher is not considered. In this preliminary stage addressed toward a better understanding of the TS body image, the analysis only studies which was, if any, the best condition to reproduce some peculiar features of the body image that up to now is impossible to reproduce in its whole.

The information used for the CD test are based on scientific results demonstrating that the TS enveloped a dead man $[9,10]$, that the body image was formed by a radiation $[1,5,10]$ and the most probable source of radiation was of electric type [10, 12-15].

The overall arrangement of the electrode system was aimed at producing a severe corona effect on the surface of a metallized manikin. The reason for the use of this metallized manikin is that under electrostatic up to radiating conditions any biological body externally behaves as perfect conductor, or nearly so. In other words, despite of the poorly conducting properties of the outer skin, as well as the inner fatty tissues and bones, there are extensive arguments leading to state that the surrounding and surface field pattern and magnitude slightly change if the real body is replaced by a metallic model $[16,17]$.

Corona Discharge (CD) manifests as a localized dielectric breakdown in a relatively small air volume under high voltage stress, which does not bridge the whole space between the two electrodes. It represents in general the first step of the discharge process which would lead ultimately to the complete breakdown.

The inception occurs when the electric field is high enough to produce ionization by collision between free electrons and neutral molecules; during the process there is normally light emission, mainly in the UV range, due to atomic excitation, de-excitation and formation of chemical byproducts typically including, in an aerial medium, ozone, nitric acid and other reactive species.

Despite the relatively simple phenomena being involved, corona discharges can be very different, depending upon polarity of the applied voltage and value of the applied field [18]. 
The term itself seems to indicate a discharge which is diffused but, in fact, this is only one possible stage of the corona discharge modes. At ambient pressure this particular condition is more common in the case of electrodes with small curvature radius and tends to be unstable because it requires an uniform distribution of the current flow in the volume [19-22].

If, for some reasons, an instability arises and the current concentrates in one particular region, then the phenomenon evolves into a filamentary type of corona discharge (streamer) which can easily lead to the complete breakdown.

Differences exist also with respect to the polarity of the applied voltage because ionization is always caused by electrons and these are forced to move in increasing or decreasing fields undergoing different attachment rates.

The difficult task of arranging the electrode setup is therefore connected to the prerequisite of a very non- uniform field near the manikin for a corona discharge to be triggered and assuming, say, the glow mode.

Preliminary studies have been carried out with models of reduced scale to identify the problems related to corona formation under the particular conditions required by the experiment.

Attempts to keep the model floating between a pair of electrodes, one of them grounded proved to work only with alternating voltages; the utilization of fast rising repetitive voltage pulses (rise time of $10 \mathrm{~ns}$ and overall duration of $300 \mathrm{~ns}$ ) was considered but it was impossible to obtain peak values above $30 \mathrm{kV}$. This implied that corona could have been produced only using extremely small models.

Very high impulse voltages in the MV range - both lightning (front duration around $1 \mu \mathrm{s}$ ) and switching (front duration of several hundreds $\mu \mathrm{s}$ ) - were available but the field required to produce corona effects under these conditions is not different from that of DC voltage, with the consequence that some discharges would rather assumed an inadmissibly energetic spark mode instead of an admissible glow one. In any case it has to be considered that the action of a corona activity on any surface is cumulative and therefore single or very few pulses cannot be expected to be very effective.

A DC voltage was therefore chosen as the most reasonable possibility in consideration of the phenomena which can be envisaged for the formation of the real image. The manikin was energized and suspended between two grounded electrodes. Corona inception is controlled by a field which in turn depends on the curvature radius; under this conditions the most active zones were disseminated on the protruding surface areas of the body.

The polarity was negative and the voltage was raised up to the maximum value compatible with the withstand of the gap; the corona type was in general resembling a glow even though ostensive filamentary discharge formed somewhere.

The CD behavior is further complicated by the presence of the linen cloth used in the experiment to simulate the TS; the interaction with the discharge development can be very different, depending on its dielectric properties which are closely associated also to the presence of oil or grease used for the impregnation.

Only a partial barrier effect [23] due to the presence of a pierced sheet (linen fabric) could arise under this condition. A full barrier effect is difficult to be reproduced when use is made of a common piece of fabric as a barrier. This because the typical dissemination of discontinuities represented by the pores can easily allow moving charges to propagate through the barrier under the action of the local electric field. In some especially critical circumstances, pre-breakdown phenomena, as well as complete breakdown, are expected to occur.

There might be a number of sub-effects that are also in contrast each other as a function of the conductor-to-barrier distance: for example, for the same voltage level, when the linen is in proximity or in contact with a conducting manikin, this effect tends to increase the electric field and, therefore, to lower the discharge onset level thus increasing the intensity of the involved corona discharges.

When the linen is kept detached, the barrier effect gives rise to discharge switch-off on the inner side of the linen, and, by compensation, field intensification on the outer side. With reference to the above 

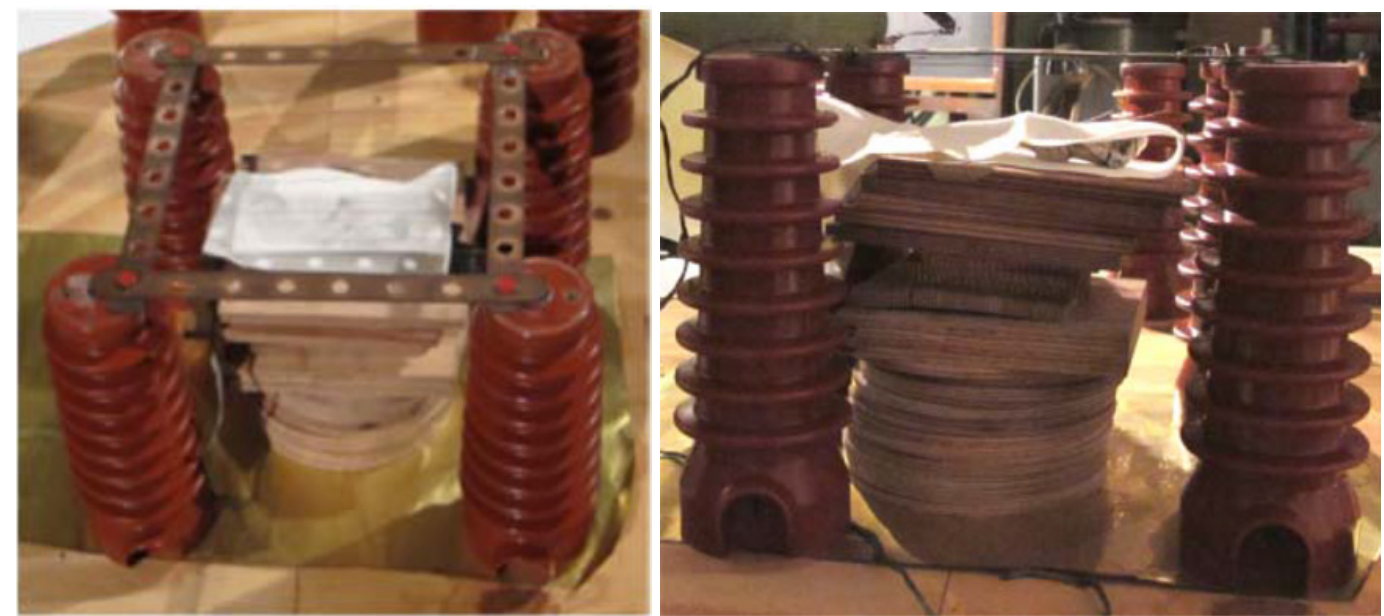

Figure 1. "Reduced-scale" setup used to test the best conditions for producing CD and images on a linen sheet.

considerations regarding a barrier made of usual fabric, an additional difficulty arises in evaluating the barrier properties of fabrics treated with those impregnating substances which are pertaining to the case study under examination.

Images of the manikin can be obtained during experiments with $\mathrm{CD}$ on the inner and/or outer side of the linen cloth adopted as a wrapping cover. If this electrostatic imaging on linen is applied to corpulent bodies like a manikin, complex models overall forming an interdisciplinary theoretical framework is required to explain the number of factors being involved.

In this context it suffices to remember that it is well established also at industrial level how CD accomplished by formation of a cold plasma can interact heavily with the superficial layers of various types of cloth, through mechanisms which include UV action, moderate heating and chemical attacks due to, as previously mentioned, the formation of ozone (O3) and other reactive by-products (e.g. nitric acid owing to the water content in air) [24].

The fiber modification is only superficial for some tens of $\mathrm{nm}$; the technique is utilized with different kinds of plasma, both at relatively low and at atmospheric pressure, even if the applied voltage is usually alternating for a better exploitation of the barrier effect.

\section{Experimental results}

Bearing in mind the experimental difficulties outlined above, also deriving by a not simple theoretical modeling of the whole electric system, the problem was tackled in two steps of increasing complexity. The first one was aimed at the determination of the best experimental conditions for CD imaging on linen sheets using a reduced-scale setup.

The second step could have been developed on a half-scale manikin after having defined the best CD conditions defined on a smaller conductive model of the TS Man.

In the first step the reduced-scale setup of Fig. 1 was composed of a rounded conductor consisting of a 11-cm long metal manikin (reported in Fig. 2) lying on an insulating wooden basement. The upper electrode system consisted of an array of six parallel metal wires each connected by a frame. The available DC generator could deliver a voltage up to $40 \mathrm{kV}$; the manikin was covered by a linen sheet similar to the TS. 

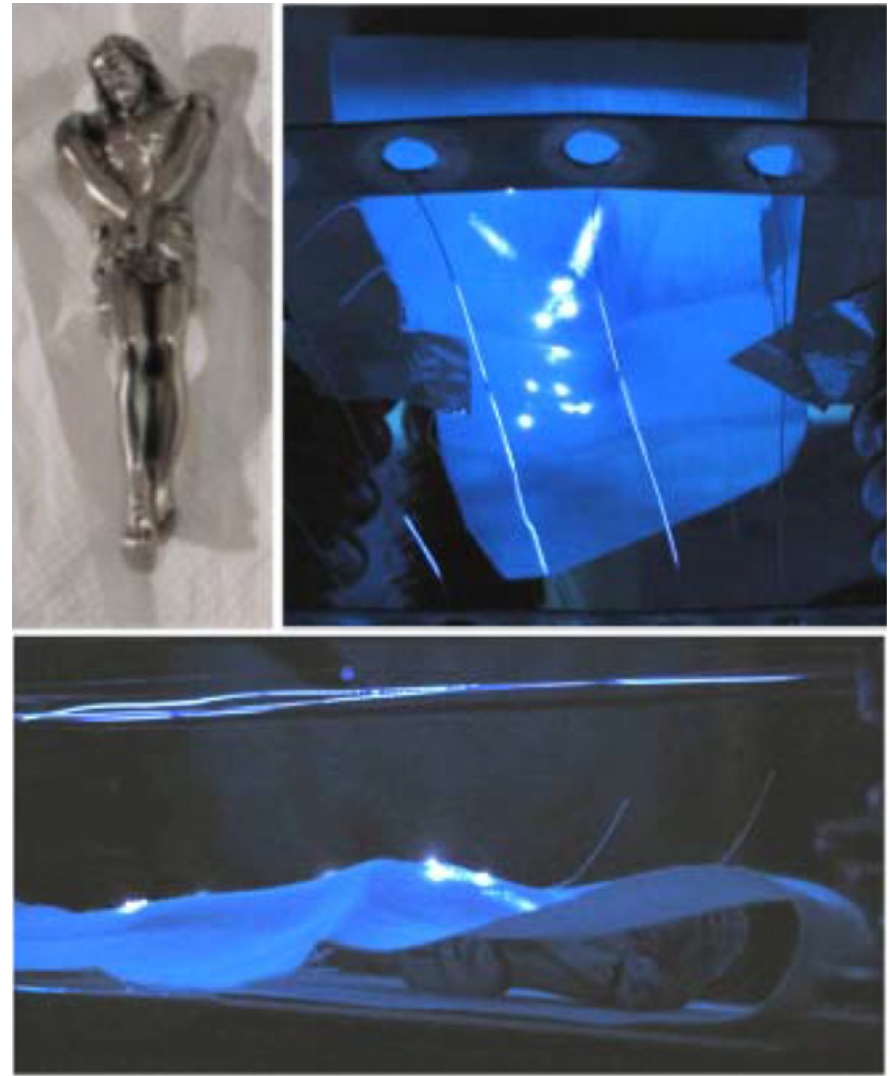

Figure 2. Reduced-scale setup producing negative CD by a $30-\mathrm{kV}$ voltage applied to a 11-cm long metallic manikin (top left). The six conductive wires (also showing CD) of the upper plate are grounded.

Many different configurations have been simulated using this setup in order to obtain the most promising image features with characteristics similar to the TS body's image. The first configuration setup was composed of:

-A. An 11-cm long metal manikin capable to give rise to $\mathrm{CD}$ activity.

-B. An upper plate connected to a potential up to $30 \mathrm{kV}$ negative $\mathrm{DC}$, or grounded, capable to become active.

-C. A grounded second conductive plate positioned below the wooden basement of the manikin.

The following configurations of the setup have been examined:

-1. A conductive manikin floating in an environment having the upper plate connected to a potential up to $30 \mathrm{kV}$ DC with negative polarity;

-2. A conductive manikin grounded with the upper plate connected to a potential up to $30 \mathrm{kV} \mathrm{DC}$ with negative polarity.

-3. A conductive manikin connected to a potential up to $30 \mathrm{kV} \mathrm{DC}$ with negative polarity and the upper plate grounded.

-4. The experiments were repeated also applying a positive voltage, but with lower success rate.

This reduced-scale setup was used to make several attempts by varying the distances between a) the upper plate (B) and the manikin (A); b) the manikin (A) and the second plate (C). 

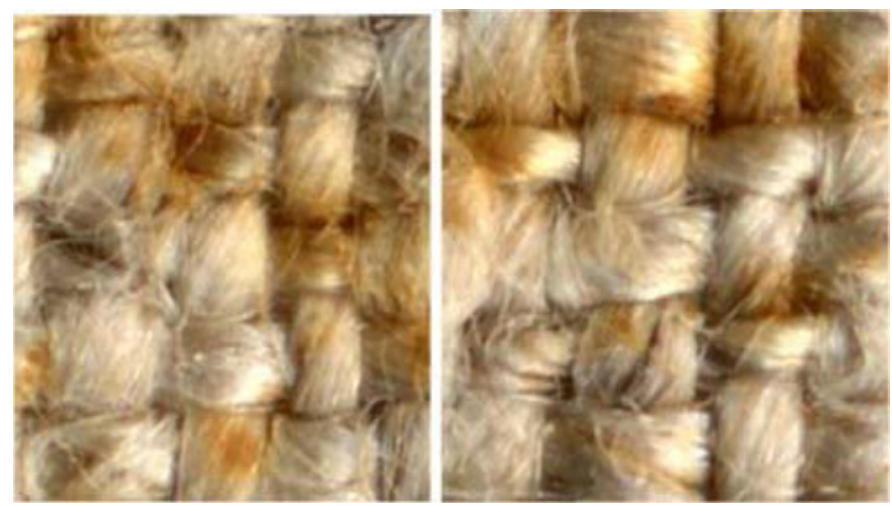

Figure 3. Experimental results of a linen fabric exposed to CD. Due to a partial barrier effect, the image is concentrated in correspondence of crevices where the electric charges are expected to concentrate.

The upper ground electrode and the basement with the manikin were positioned at a distance which has been varied from 1 to $15 \mathrm{~cm}$. This has been made in order to determine the best condition capable to produce a cloth imaging. A gap of about $4 \mathrm{~cm}$ proved to be the best one and this scaled parameter was used as a constructional reference that led to set up the final half-size scale experimental apparatus.

Other tests were performed to additionally simulate the effects of the possible presence of spices and oils daubed on the TS Man that could have influenced the generated electric field. A silicon compound (Greasil MS 354) was employed for this purpose. Nevertheless, the best TS-like image on the linen sheet was given in absence of silicon daubing.

Also this additional information was taken into account to make up the above-mentioned setup, in which case the mockup was covered by a linen sheet having no impregnating substances added.

Figure 2 shows the reduced-scale setup subject to CD activity. This is confined to the prominent locations of the manikin (arms, hands and legs) on which a TS-like cloth was lied down without rolling it up. This description is relative to Configuration (A-3), in which the human model was connected to a negative DC potential of $30 \mathrm{kV}$.

The resulting glow corona, visible in the setup of Fig. 2, seems to be affected by some sort of barrier effect [23] exerted by the linen sheet. The charging of the cover due to the free carriers results in the lowering of the corona onset level because of the electric field increase on the conductor for a given applied voltage.

On the other hand, the close vicinity of the fabric to the active conducting manikin prevents strong charge accumu-lation on the inner side of the sheet, which is at the same polarity as the deposited charges, to allow pre-breakdown events to happen.

The hypothesis of the presence of something like a barrier effect produced by an intense electric field also in correspondence of the of the TS body image is sustained by a particular feature reported as follows in Ref. [7]: “... the color of the image areas has a discontinuous distribution along the yarn (threads) of the cloth: striations are evident. ... from the photographs a color concentration can be detected in correspondence to crevices where two or three yarns cross each other...".

The partial barrier effect induced by a pierced sheet, as in fact the linen fabric is, produces a charge concentration in correspondence of the holes that therefore become more colored than the surrounding threads. This fact has been detected during the CD experiments, see Fig. 3.

Experimental set-up (3) proved to be the only one capable to produce well detectable images on the linen sheet enveloping the manikin. Therefore this was the configuration selected for the second step in which a half-scale manikin was employed. 


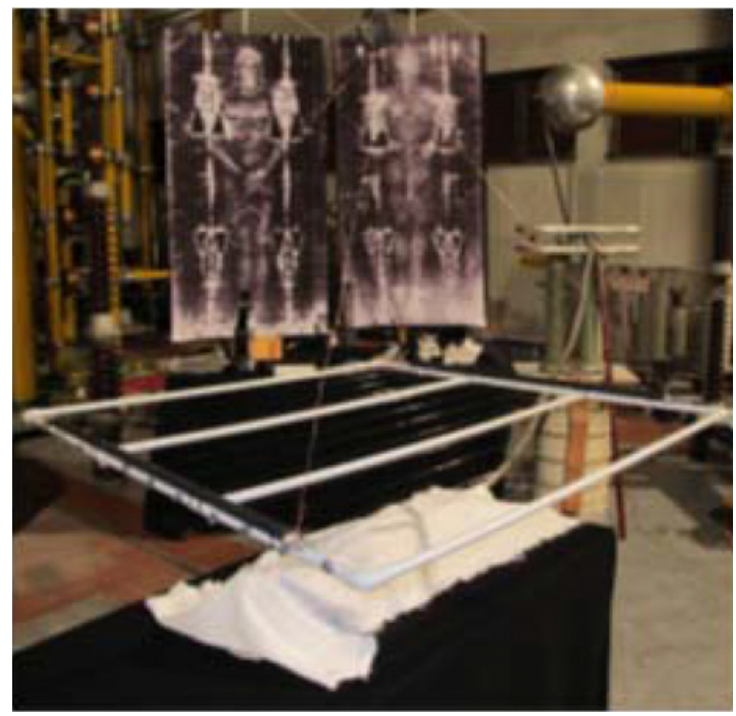

Figure 4. Experimental set-up for the half-scale conductive sculpture enveloped in a TS-like fabric subjected to a CD generated by $300 \mathrm{kV}$.

The half-scale experiment shown in Fig. 4 was set up by employing a terra-cotta sculpture of a human subject postured in a way very similar to that reconstructed from the TS image. It was covered with a conductive paint and then enveloped in a TS-like fabric. This system was interposed between two electrodes at ground potential.

When the conducting mockup was raised to a voltage of $300 \mathrm{kV}$, vivid $\mathrm{CD}$ appeared. The upper electrode at ground potential was made with a row of thin wires to increase the upper limit of the applied voltage and to obtain additional ionization, thus producing a strong ion flow surrounding the manikin.

Previous laboratory tests showed that DC sources of the order of $300 \mathrm{kV}$ are needed to reproduce electrostatic images on linen fabrics, enveloping rounded-off conducting surfaces, that produce partial barrier effects.

The CD image shown in Fig. 5 was obtained after 24 hours of CD exposition with subsequent artificial aging produced by ironing for about $60 \mathrm{~s}$ at a temperature of $180 \pm 15^{\circ} \mathrm{C}$.

It must be here added that Configuration (1) relevant to the smaller setup was based on a hypothesis of a field distribution reported in Refs. [24, 25] where imaging has been numerically obtained by an exogenous and uniform electric field applied to a discharged and floating body [24].

As confirmed by other numerical simulations [26] and by both a theoretical model and experiments [25], the radial electric field on the surface of the conductor in this case (see Configuration 1) is described by the Lambert's cosine law, whereas Experimental set-up (3) produces a nearly radial and constant field.

The unsuccessful results produced by these tests using Configuration (1) has been explained in [24] by the lack of a necessary pre-ionized gas as instead it is the case for a radon-prone ambient under special circumstances.

\section{Image distortion}

The Turin The experimental result of Fig. 5 shows an evident distortion that it is not typical of the TS body image, especially in correspondence of the upper legs and face. Even if it was demonstrated that 


\section{SHS Web of Conferences}
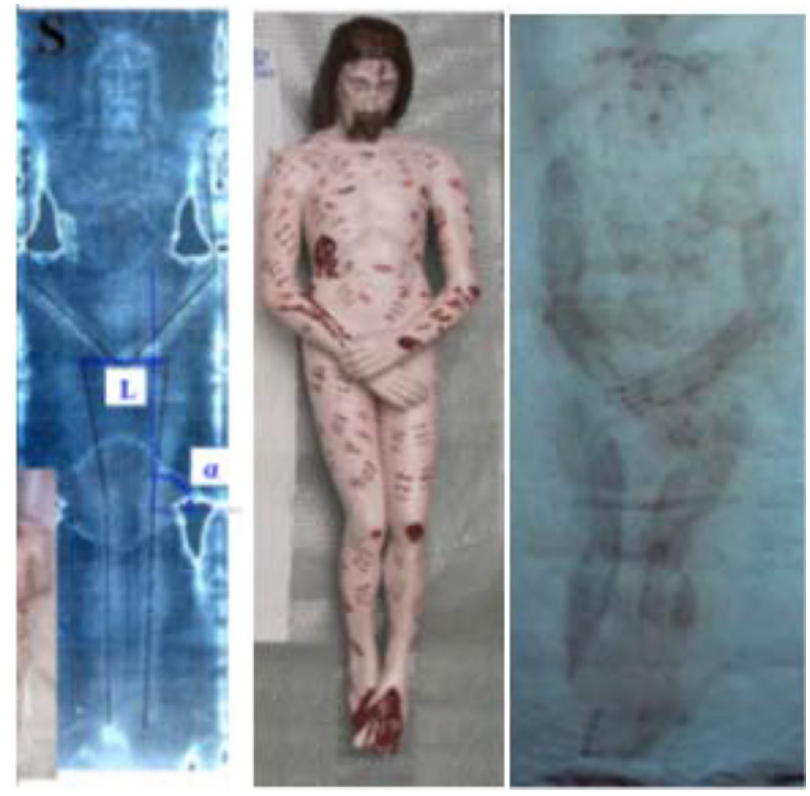

Figure 5. The negative image of the TS (left) compared with a half-scale TS-like manikin (central) and the image (right) obtained on the external surface of an ironed TS-like sheet illuminated by UV. This previously enveloped a half-scale conductive manikin connected to dc potential of $300 \mathrm{kV}$ for 24 hours.

the TS image presents distortion of pseudo-cylindrical type produced by the TS that wrapped a human body [28-30], this distortion is not so relevant as that resulted in the CD experiment.

It is well known that the complex phenomena related to the CD distribution law around a variously postured and corpulent body is not simple to study. Therefore, preference has been expressed for the described experimental approach.

A half-scale semi-transparent copy of the TS has been printed and then superimposed on the manikin to verify the correspondence within a tolerance of $1 \mathrm{~cm}$ (Fig. 6). An image portion restricted to the hands and legs of Fig. 5 was selected for detection because this detail is better appreciable than those placed elsewhere. Special attention was paid for the leg contour, that was full arrowed (for highlighting purposes) on the copy surface, before being positioned above the manikin legs.

To strengthen the above approach, note that there is no consensus elsewhere on the very mechanism accounting for the TS body image formation. Additionally, the imaging in question rather turns out to be a non-linear phenomenon not easy to interpret so far.

Notwithstanding these drawbacks, overlapping the semi-transparent copy of the TS on the manikin tentatively allowed establishment of those manikin leg's areas which would have been actually implied in the image formation.

It is worth considering that such a simple but meaningful method has the merit of even roughly appreciate the locations of major electrostatic stress (see the companion paper [31]). This merely because the latter is confined on those protruding parts of the body which in fact happen to be attached to a covering sheet, provided it is laid smoothly down on the body, throughout.

Furthermore, under the same circumstances, the sheet tends to remain detached from the depressions of the body surface nearly in the place where the electrostatic activity could turn off.

As a result of the described overlapping, the leg contour of the TS-like sheet corresponds to a position on the manikin's leg dictated by the intersection of the leg surface with the line inclined of $45^{\circ}$, or thereabouts, according to the polar representation in Fig. 7. 


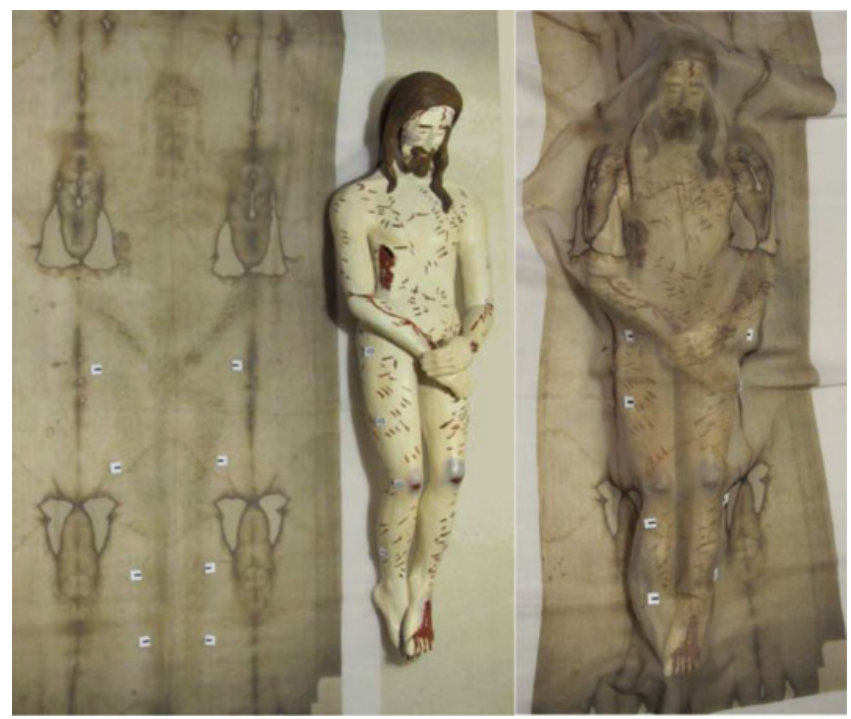

Figure 6. Left: half-scale semi-transparent copy of the TS with the leg contours highlighted and the corresponding manikin used for the distortion analysis. Right: TS-like manikin wrapped with a semi-transparent sheet.

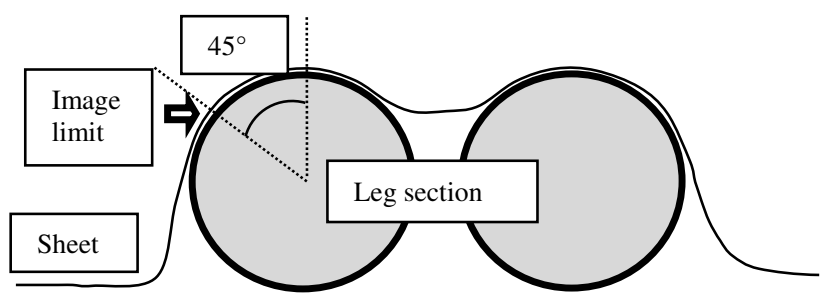

Figure 7. Cross-sectional view of the legs covered by a draping sheet. The border arrow indicates the peripheral contour of the body image impressed on the sheet.

This information has therefore been used to approximately identify the position on the manikin surface area that impressed an image on the TS. This area is instead evidenced by border arrows as opposed to the full arrows adopted for the semi-transparent copy mentioned above.

Once the image borders were traced, in agreement with the described simple rule, both on the semitransparent copy of the TS and on the manikin, a whole-body image inspection was performed by directly overlapping the TS copy on the manikin.

When the semi-transparent sheet wrapped the manikin, an attractive fact came to the investigators' attention: both the arrows of the manikin and the sheet are almost coincident along the legs lines, whereas a nearly 4-cm spacing is detected between the corresponding arrow-shaped full and border marks disseminated on the hand area of both sides of the body, see Fig. 8. It has to be deduced from all this that the presence of the hands increases the pseudo-cylindrical distortion of the sheet around the manikin, thus producing the disfiguration of Fig. 5.

As an immediate consequence, the adopted method leading to the angular shift, depicted in Fig. 7, should need to be revised at the light of the results shown in Fig. 8. This could be made by taking the additional distortion caused by nearby human parts, like the hands also involved in the wrapping, into 


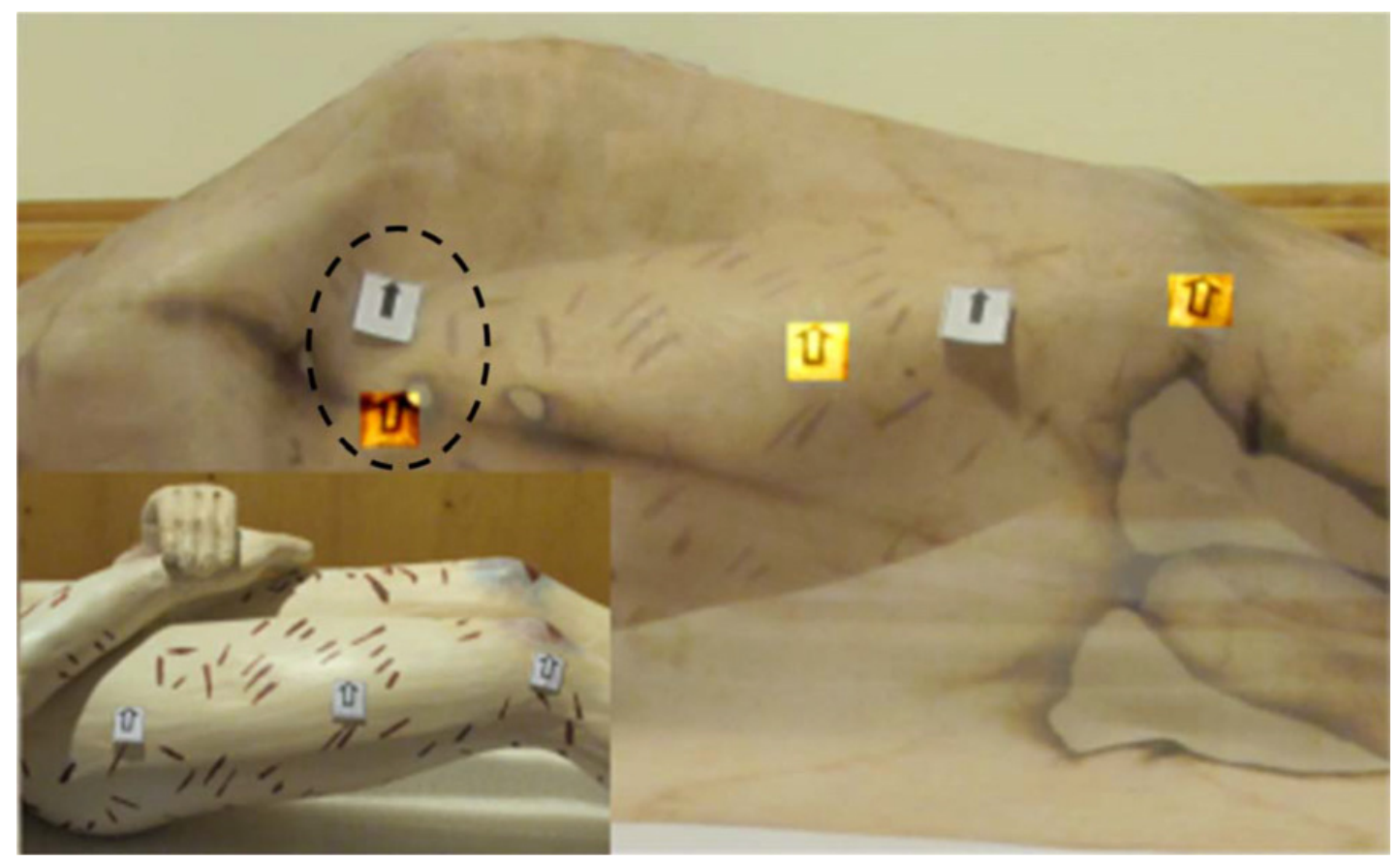

Figure 8. Right-thigh area of a wrapping semi-transparent sheet with an impressed TS image. The displacement of about $4 \mathrm{~cm}$ of the sheet-arrow with respect to the corresponding manikin-arrow in the hand area (circled) is clearly perceivable, thus explaining the relevant distortion on the experimental CD image.

due account. Once again, the raised similarity with the mutual electrostatic influence between nearby conducting protrusions can be appreciated. This result leads to the following considerations.

1. In order to reduce the distortion on the CD-originated experimental image of Fig. 5, a different way of enveloping the manikin into the sheet by stretching it can be investigated.

2. As previously reported, the TS body image presents some pseudo-cylindrical distortions that are however less pronounced than those obtained by a cloth tightly wrapping the manikin.

3. The distance of legs in the experimental CD image of Fig. 5, that appears greater than the same distance of the TS legs, is in favor of a flatter surface enveloping the manikin.

An experimental verification was performed looking at the inner manikin through the half-scale semitransparent copy of the TS laying according to different draping configurations around the manikin itself.

The approach leads to recommend that the sheet is enveloped around the manikin maintaining it flatter than the configuration of Fig. 5, thus preventing either a tightly wrapping or a rigidly flat coverage. Future experiments reproducing this last draping configuration should be performed to verify this deduction.

Of course, the above advices need to be applied to the experimental example here treated in which the conducting body is a charged one, namely in which the conductor itself is the source of the electric field triggering the image process.

Provided that the above endogenous field instead becomes an exogenous one, namely that the body identifies with a discharged conductor introduced into a background electric field, then the electrostatic scenario so changes [24] that the above issues on the wrapping method must be revised. 


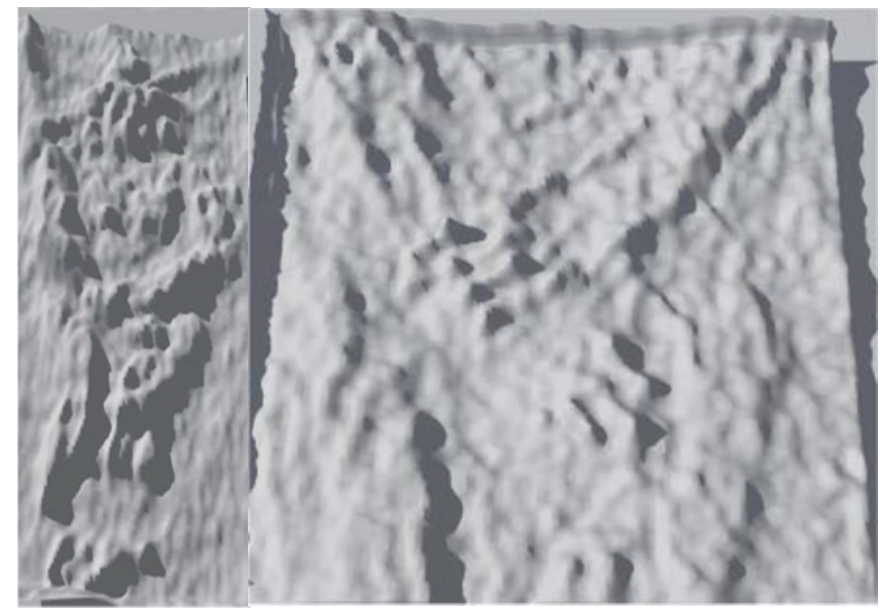

Figure 9. 3D processing of the experimental image obtained using $\mathrm{CD}$ with a detail of hands on the right.

In addition, efforts can be made to experimentally reproduce in laboratory electrostatic images on human shaped floating conductors. Under the described circumstances, the unsuccessful imaging seems determined by the practical difficulties that arise in triggering discharges in normally ionized ambient air.

\section{Discussion}

Figure 5 demonstrates that it is possible to obtain a human image on a TS-like linen sheet enveloping a conductive manikin subjected to $\mathrm{CD}$. This image has many features very similar to those detected on the TS, especially at microscopic level. A suitable description is found in [7, 12], where the typical superficiality both at fabric and at fiber level is emphasized.

A first comparative analysis performed at macroscopic level between the electrostatic and TS images evidences likenesses and differences summarized as follows.

-1. Both the images are of negative type and some 3D features are detectable on the electrostatic image, see Fig. 9.

-2. The resolution of the CD image is in general comparable with that of the TS, even if the face is expected to be defined better after properly assuming a draping configuration for the cover.

-3. As discussed in Section IV, the experimental CD image is more distorted than the TS one; this pseudo-cylindrical distortion is due to the adopted draping configuration, a drawback especially appreciable in correspondence of the hands.

-4. Both the TS and the CD-related images show marks of double superficiality [8], but while the TS image is better perceivable on the inner side of the cover, namely the one in contact with the human body, the CD experimental image is conversely better impressed on the external surface. This fact must be better studied in reference to the electric field conditions.

In addition, it seems interesting to observe an imaging effect also in some areas where the body-to-cloth gap is relatively wide (about $5 \mathrm{~cm}$ in the half-scale model) as it happens to be in correspondence of the belly.

As reported in [29], some scholar supposed that voltage of nearly $60 \mathrm{MV}$ was to necessary for a glow thickness to impress an image also in correspondence of the belly, a crucial location where the body-sheet distance can attain about $10 \mathrm{~cm}$. 
Even though the present experiment has been made in a half-scale model, it is clear that much lower potentials can be able to color the linen sheet also when the body-cloth distance is of some centimeters (just in correspondence of belly-hands area). This result demonstrates that even potentials much lower than $60 \mathrm{MV}$ are however large enough to impress a human image on a wrapping cloth.

The non-linear phenomena [20] associated to CD, and concurring other local effects, can be responsible for imaging without making recourse to exceedingly high voltages. For example when the linen is detached from the body, the partial barrier effect gives rise to discharge switch-off on the inner side of the linen, and, by compensation, field intensification on the outer side. Also the ionic drift [27] and the consequent accumulation in the volume between the manikin and the cloth could be responsible for some imaging of the covering cloth where the body-cloth gap is relevant.

The evident pseudo-cylindrical distortion of the CD image seems to be in favor of a TS draping configuration different from that simulated during the CD experiment; in fact the TS image appears produced by a flatter sheet than one corresponding to a tight wrapping. But accounting for an almost undistorted imaging, like that of the TS, is quite a complicated matter requiring multidisciplinary efforts as those involving electrostatics, computer vision and chemistry to name a few.

Also the double superficiality of the experimental body image, opposed in position (in-out) with respect to that detected on the TS [8] must be better studied also in reference to the electrode setup.

A possible explanation of the obtained result can be the following: the charge accumulation on the linen side facing the distanced conductive manikin becomes the source of additional field enhancement on the outer side. As a result, a phenomenon like photoionization can give rise to outward discharges, thus causing marks detectable on the outer side of the linen sheet.

Also a type of partial barrier effect involving a multi-pore sheet as a cover for the manikin needs additional investigation in the next future.

\section{Conclusive remarks}

A tentative to reproduce the TS body image using CD has been done in the high-voltage laboratory of the University of Padua using a linen cloth in which a half-scale conductive manikin of a man was enveloped before being suspended from ground and connected through the feet to a $300 \mathrm{kV}$ DC supply.

A TS-like image has been obtained using a set-up in which the manikin was covered by a sheet and positioned below a grounded electrode. This resulting image is strictly connected to the electric field distribution of the environment where the manikin was introduced to impress the body image.

Many features, peculiar of the TS image, have been reproduced especially at microscopic level. Some differences at macroscopic level like the pseudo-cylindrical distortions and the image impression outside the enveloped manikin are a useful basis for future studies regarding the draping configuration of the TS Man.

Instead no images derived from a floating disposition of the manikin have been produced. The fact that no images have been obtained when $\mathrm{CD}$ was generated by an exogenous electric field poses some difficulties in the application of a hypothesis based on this assumption, unless recourse is made to a pre-ionized gaseous ambient as in the case of a radon-prone ambient.

From experimental data aimed at evaluating distortion effects, it derives that the TS was not tightly wrapping the human body during the exposition to $\mathrm{CD}$. Therefore a rather stretched cover is envisioned to be suitable to explain successful image performance especially in correspondence of the hands.

Potentials remarkably lower than $60 \mathrm{MV}$, as proposed in the past by some scholar to produce a TS-like image, are sufficient and this fact can also be correlated to the ionic drift.

The results of the described experiments on one hand show how much difficult is, if not impossible, to reproduce the TS body image in a laboratory, but, on the other hand, offer substantial arguments to claim that the hypothesis based on an electrostatic mechanism is, among others, the best one to account for the very peculiar image under examination. 
It seems appropriate here to observe that the experimental configuration capable to produce images on a linen sheet is not against the supposed condition (up to now neglected by the authors) of the body of Jesus Christ put in the rocky sepulcher. Both the rocky basement, on which the corpse imaginatively lies and ceiling of the sepulcher assume the role of an equipotential conducting interface because of their moist conditions. The pair of grounded conductors adopted in the laboratory are representative of the conducting boundaries at ground potential whose gap could roughly represent the sepulcher height.

This study is one of the results obtained from the financial support of the University Research Project-Padova, Italy2009 \#CPDA099244 entitled: "Multidisciplinary analysis applied to the Shroud of Turin: a study of body image, of possible environmental pollution and of microparticles characterizing the linen fabric".

\section{References}

[1] Jumper, E.J., Adler A.D., Jackson J.P., Pellicori S.F., Heller J.H., Druzik J.R., "A comprehensive examination of the various stains and images on the Shroud of Turin", Archaeological Chemistry III, ACS Advances in Chemistry n ${ }^{\circ}$ 205, J.B. Lambert, Editor, Chapter 22, American Chemical Society, Washington D.C., 1984, pp. 447-476

[2] Adler A., Shroud Spectrum Int. Special Issue, Effatà Editrice, Torino, Italy, (2002)

[3] Vignon P., "The Shroud of Christ", E. P. Dutton \& Co. New York 1902

[4] Schwalbe, L.A., Rogers R.N., "Physics and chemistry of the Shroud of Turin, a summary of the 1978 investigation", Analytica Chimica Acta, Vol. 135, 1982, pp. 3-49

[5] J.P. Jackson, E.J. Jumper, and W.R. Ercoline, "Correlation of image intensity on the Turin Shroud with the 3-D structure of a human body shape", Appl. Opt. 23, 2244-2270 (1984)

[6] E. Marinelli, "La Sindone, un'immagine 'impossibile"', Ed. San Paolo, Cinisello Balsamo (MI) 1996 e 1998

[7] G. Fanti, J.A. Botella, P. Di Lazzaro, T. Heimburger, R. Schneider, N. Svensson,” Microscopic and Macroscopic Characteristics of the Shroud of Turin Image Superficiality", J. of Imaging Sci. Technol., 54 No. 4, p. 040201-1/8, (2010)

[8] G. Fanti and R. Maggiolo, "The double superficiality of the frontal image of the Turin Shroud", J. Opt. A, Pure Appl. Opt. 6, 491-503 (2004)

[9] G. Fanti, J.A. Botella, F. Crosilla, F. Lattarulo, N. Svensson, R. Schneider, A.D. Whanger "List of Evidences of the Turin Shroud", Int. Workshop on the Scientific Approach to the Acheiropoietos Images, ENEA Research Center of Frascati (Italy), 4-5-6 May 2010

[10] G. Fanti, "Hypotheses regarding the formation of the body image on the Turin Shroud. A critical compendium", J. of Imaging Sci. Technol., Vol. 55, No. 6, p. 060507 (Dec 22 2011)

[11] F. Lattarulo, "TS image explained by a sismo-electric process" (translated from Italian). III Congresso internazionale di studi sulla Sindone, Torino, June 5-7 1998, pp. 334-346

[12] G. Fanti, "Can a Corona Discharge Explain the Body Image of the Turin Shroud?", Journal of Imaging Science and Technology, Vol. 54, No. 2, March/April 2010, p. 020508-1/10

[13] G. Fanti, F. Lattarulo, and O. Scheuermann, "Body image formation hypotheses based on corona discharge", Third Dallas International Conference on the Shroud of Turin: Dallas, Texas, (2005)

[14] A. Whanger and M. Whanger, The Shroud of Turin: An Adventure of Discovery (Providence House Publishers, Franklin, TN, 1998)

[15] G.B. Judica Cordiglia, "La sindone immagine elettrostatica?", in La Sindone, Nuovi Studi e Ricerche, Atti del III Congresso Nazionale di Studi sulla Sindone (Edizione Paoline, Cinisello Balsamo, Italy (1986) pp. 313-327 


\section{SHS Web of Conferences}

[16] V. Amoruso and F. Lattarulo, Diakoptic Approach to EMC Problems Involving the Human Body”, in: Electromagnetic Compatibility in Power Systems, F. Lattarulo Editor, Elsevier, Oxford (UK), 2007, pp. 133-164

[17] F. Lattarulo, Specific Absorption Rate (SAR) Revisited for the Ellipsoidal Model of a Human Body, Engineering Analysis with Boundary Elements (Special Issue on Special issue on: Boundary Elements and Other Reduction Methods in Bioelectromagnetics), vol. 49, 2014, pp. 31-36

[18] L.B. Loeb: "Electrical coronas - Their Basic Physical Mechanisms", University of California Press, 1965

[19] J.D. Stephenson, "Corona and spark discharge in gases", Electrical Engineers, Journal of the Institution of (Volume: 73, Issue: 439) (1933), pp. 69-82

[20] A.A. Belevtsev "Modern Theory Of Corona Discharge" Proc. 3rd Int. Conf. Properties and Applications of Dielectric Materials (1991), Tokyo, Japan

[21] H.X. Liu, S.W. Ming, W. Lei, "Measurement and analysis of Electromagnetic Fields Radiated by Corona Discharge", Int. Symp. Electromagnetic Compatibility, EMC 2007. (2007), pp. 31-34

[22] V.I. Popkov, "On the Theorv of Corona Discharge in Gas under Constant Voltage", Izv.Akad.Nauk SSSR, Otd. Techn. Nauk, No 5, pp. 664-674 (1953)

[23] M. Abdel-Salam, H. Singer and A. Ahmed, "The barrier effect on the onset voltage of negative corona in air" J. Phys. D: Appl. Phys., vol. 30, 1997, pp. 1017-1028

[24] Amoruso V, Lattarulo F., "A physicochemical interpretation of the Turin Shroud imaging", Scientific Research and Essays, Vol. 7 (29), pp. 2254-2579 (2012)

[25] F. Roman, V. Cooray and V. Scuka, "Corona from floating electrodes", Journal of Electrostatics, vol. 37, 1996, pp. 67-78

[26] G. Fanti, L. Matordes, V. Amoruso, M. Bullo, F. Lattarulo, G. Pesavento, "Numerical Simulation of a Human Body Subjected to Electrostatic Fields for Study of the Turin Shroud Body Image", COMSOL Conference, Milan, Italy, 10-12 October 2012

[27] Amoruso V., Lattarulo F., "Filamentary Flow: Theory and Experiments", Wiley-IEEE Press, 2014

[28] Ercoline, W.R., Downs R.C. Jr., Jackson J.P., "Examination of the Turin Shroud for image distortions", IEEE 1982 Proceedings of the International Conference on Cybernetics and Society, (1982), Seattle, pp. 576-579

[29] G. Fanti, "Sindone, una sfida alla scienza moderna”, Ed. Aracne, Italy (2009)

[30] G. Fanti, R. Basso: "Optics Research Applied to the Turin Shroud: Past, Present and Future" Chapter 1 of "Optics Research Trends" (pp. 1-60), Editor: Paul V. Gallico, Publisher Nova Science Pub Inc., USA, (2007)

[31] G. De Liso, G. Fanti, F. Lattarulo, "Turin Shroud-like Electric Imaging Connected to Earthquakes", 2014 IEEE-ATSI Workshop, Bari, Italy 